\title{
Comunicação científica e gestão do conhecimento: enlaces conceituais para a fundamentação da gestão do conhecimento científico no contexto de universidades
}

\author{
Scientific communication and knowledge management: \\ conceptual correlations for the foundation of scientific \\ knowledge management in the academic context
}

Fernando César Lima LEITE'

\begin{abstract}
RESUMO
Este artigo, produto de pesquisa teórica, tem por objetivo sinalizar as similaridades teóricas entre a abordagem da gestão do conhecimento e a da comunicação científica. $O$ esclarecimento dos pontos de convergência entre uma e outra abordagem justifica-se pela necessidade de fundamentação conceitual para os estudos de gestão do conhecimento científico no ambiente acadêmico, visto que a literatura existente, até o presente momento, não considera as especificidades desse contexto, nem tão pouco a natureza da produção e da comunicação desse tipo de conhecimento. As reflexões consideram, inicialmente, a comunicação e gestão do conhecimento do ponto de vista das organizações empresariais, elaborando modelo teórico que ilustra a relação entre comunicação, cultura e gestão do conhecimento. Em um segundo momento, com base em reflexões teóricas, direciona a discussão para o ambiente acadêmico, considerando no modelo a comunicação científica, a cultura científica e a gestão do conhecimento nesse meio. Em seguida, explora as similaridades entre gestão do conhecimento e a comunicação científica, com base na comparação entre os processos de cada uma das abordagens. Conclui destacando os processos de comunicação científica como camada essencial e indispensável à gestão do conhecimento científico no contexto de universidades.
\end{abstract}

Palavras-chave: gestão do conhecimento; comunicação científica; gestão do conhecimento científico; gestão do conhecimento em universidades.

\begin{abstract}
A B S T R A C T
This article, product of theoretical research, has as goal to signal the theoretical similarities between the approaches to knowledge management and to scientific communication. The clarification of the convergence points between such approaches is justified by the necessity of conceptual foundation for the studies on scientific knowledge management in the academic environment, since the specialized literature until the present moment seems not to consider the specificities within this context, or even the characteristics of the production and the communication of this type of knowledge. These reflections consider initially, the communication and knowledge management from the point of view of the enterprise organizations, preparing a theoretical model that illustrates the relationship between communication, culture and knowledge management. In a second moment, on the basis of theoretical reflections, it directs the discussion to the academic environment, considering, in the model, the scientific communication, the scientific culture and the knowledge management. After that, it explores the similarities
\end{abstract}

\footnotetext{
1 Pesquisador Colaborador, Universidade de Brasília. Campus Universitário Darcy Ribeiro, Asa Norte, 70910-900, Brasília, DF. E-mail: <fernandoc@unb.br>.

Recebido em 25/10/2006 e aceito para publicação em 29/5/2007.
}

TransInformação, Campinas, 19(2):139-151, maio/ago., 2007 
between knowledge management and the scientific communication, based on a comparison between the processes involved in both approaches. The conclusion points out to the processes of scientific communication as an essential and indispensable layer to the management of scientific knowledge within the university context.

Keywords: knowledge management; scientific communication; scientific knowledge management; knowledge management in universities.

\section{N T RO D U ÇÃ O}

A gestão do conhecimento surge e tem como habitat natural as organizações empresariais e, como objeto, o conhecimento organizacional. Contudo, existem outros contextos nos quais há atividades intensivas em conhecimento como, por exemplo, o ambiente acadêmico. É possível afirmar que a universidade, como o celeiro principal da produção do conhecimento científico, constitui um campo fértil para a aplicação e o estudo da gestão do conhecimento. Esse pressuposto está fundamentado em duas principais questões. Primeiro, as atividades da universidade estão diretamente relacionadas com a produção e a comunicação do conhecimento científico, seja por meio da pesquisa científica, seja por meio do processo de ensinoaprendizagem. O segundo argumento, complementar ao anterior, diz respeito ao fato de que a universidade, por constituir um sistema científico maior, está envolvida por uma cultura científica que preza e privilegia o compartilhamento do conhecimento que é constantemente produzido. Ou seja, no contexto científico, quanto maior visibilidade o trabalho de um pesquisador alcançar, mais chances de ser citado ele terá. Por conseqüência, mais prestígio e reconhecimento ao pesquisador, o que, por sua vez, será revertido em uma espécie de 'moeda' que garante maiores chances, por exemplo, de obtenção de financiamento para pesquisas.

A comunicação é recorrentemente mencionada como um elemento essencial para a gestão do conhecimento organizacional. Contudo, ainda pouco se tem dito sobre a relação entre a comunicação científica e a gestão do conhecimento natural do ambiente acadêmico, o conhecimento científico. Trata-se obviamente de processos de natureza distinta, existindo uma significativa diferença entre os processos de comunicação e de gestão do conhecimento nos dois contextos, uma vez que, no ambiente acadêmico existe pré-disposição, por parte dos pesquisadores, para a produção e compartilhamento intensivo do conhecimento científico.

Este artigo, produto de pesquisa teórica ${ }^{2}$, tem por objetivo sinalizar as similaridades teóricas entre as abordagens da gestão do conhecimento e da comunicação científica. $\bigcirc$ esclarecimento dos pontos de convergência entre uma e outra abordagem justificase pela necessidade de fundamentação conceitual para estudos de gestão do conhecimento científico no ambiente acadêmico, visto que a literatura existente, até o presente momento, não considera as especificidades desse contexto, nem tampouco a natureza da produção e da comunicação desse tipo de conhecimento.

Inicialmente, as reflexões consideram a comunicação e gestão do conhecimento do ponto de vista das organizações empresariais. Em um segundo momento, com base em reflexões teóricas, direciona a discussão para o ambiente acadêmico, considerando a comunicação científica, a cultura científica e a gestão do conhecimento desse meio. Logo em seguida, explora as similaridades entre a gestão do conhecimento e a comunicação científica com base na comparação entre os processos de cada uma das abordagens.

\section{Processos de comunicação, cultura e gestão do conhecimento}

A análise da literatura sobre comunicação e gestão do conhecimento organizacional oferece uma série de argumentos que sugerem a necessidade de se levar em consideração a comunicação como elemento chave da gestão do conhecimento. Ash (2000) afirma que uma comunicação efetiva é essencial para qualquer programa de gestão do conhecimento. Da mesma forma, Jensen (1998), segundo trecho publicado pela Knowledge Management Review ${ }^{3}$, afirma que a

2 LEITE, F. C. L. Gestão do conhecimento científico no contexto acadêmico: proposta de um modelo conceitual. 2006 . Dissertação (Mestrado em Ciência da Informação) - Universidade de Brasília. Brasília.

3 "Studies have shown that tacit knowledge [what we know but not yet 'captured' in script -or database-format] is still best transferred during...communication. It's estimated that $50 \%$ to $95 \%$ of transferred knowledge...occurs during oral communication". 
comunicação e a gestão do conhecimento estão ligadas, sendo que a diferença entre uma e outra é que a gestão do conhecimento é um pouco mais disciplinada em capturar, organizar e rastrear o que nós necessitamos para tomar decisões, enquanto que a comunicação está mais voltada para as trocas. Sharp (2003) e Martensson (2000) enumeram elementos críticos para o sucesso da gestão do conhecimento e dentre eles os autores incluem a comunicação. Tais afirmações nos levam a pensar em uma relação de complementaridade e interdependência entre as duas práticas. Isso por que, por um lado, a gestão do conhecimento disciplina, sistematiza e torna mais efetivos os processos de comunicação. Por outro, a comunicação permite que a gestão do conhecimento seja viabilizada, pois possibilita, dentre outros processos, a interação entre indivíduos e, por conseqüência, a criação, troca e compartilhamento do conhecimento.

A partir de reflexões e informações extraídas ao longo da análise da literatura específica relacionada aos aspectos teóricos sobre informação e conhecimento, dos processos de comunicação e da gestão do conhecimento (Leite, 2006), é possível sugerir, como parte dos resultados do estudo ${ }^{4}$, que, em qualquer ambiente, a relação entre a comunicação, o contexto/cultura e a gestão do conhecimento pode ser representada no modelo ilustrado na Figura 1.

Observam-se no modelo dois elementos que podem ser considerados como estruturais em qualquer sistema de gestão do conhecimento: os processos de comunicação e o contexto/cultura da organização. Pouco se tem explorado efetivamente a comunicação, do ponto de vista de seus processos, como um elemento da gestão do conhecimento. Muito embora a questão da comunicação seja ressaltada como importante, especialmente a comunicação organizacional, parecem ser raras as iniciativas de gestão do conhecimento que, de fato, levam em consideração os processos de comunicação, e não somente as tecnologias, como uma camada essencial de projetos de gestão do conhecimento. Ao considerar a comunicação apenas sob o aspecto das tecnologias, o discurso da gestão do conhecimento torna-se reducionista. De outro modo,

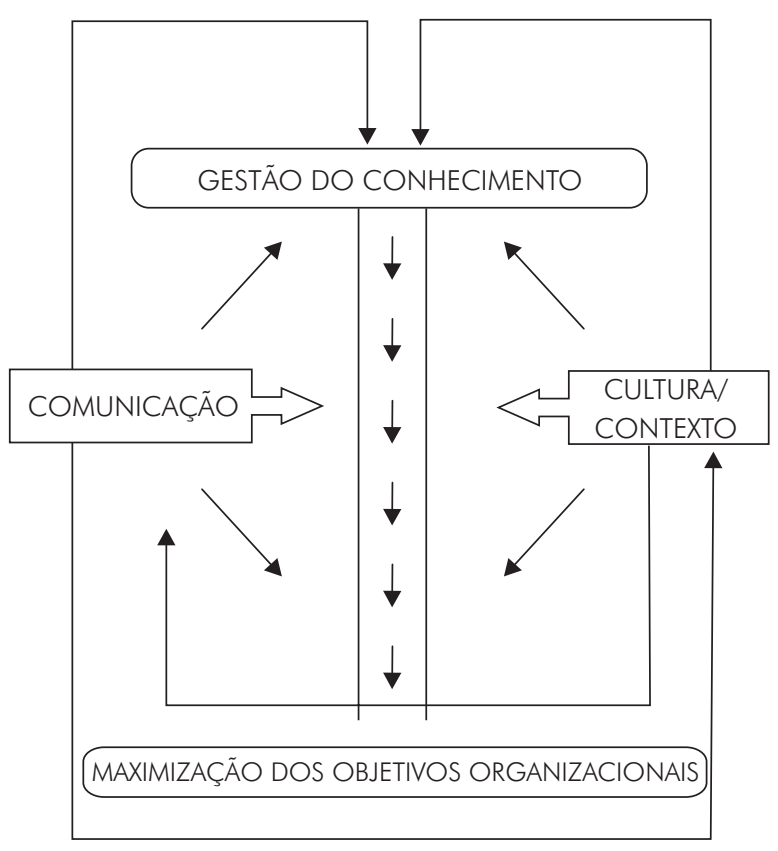

141

Figura 1. Modelo que ilustra as relações entre processos de comunicação, cultura e gestão do conhecimento.

Fonte: Leite (2006).

a comunicação deve ser vista como um elemento de forte influência no desempenho das atividades organizacionais e subjacente a todas elas, sobretudo na gestão do conhecimento, uma vez que a comunicação é responsável pelo compartilhamento do conhecimento e da aprendizagem e pela difusão da cultura da organização.

Ao se discutir a gestão do conhecimento e o processo de comunicação propriamente dito, é inevitável inserir no debate as peculiaridades do contexto e da cultura do ambiente no qual a comunicação está sendo estudada. Nesse caso, a cultura é entendida como um

Padrão de pressuposições básicas partilhadas aprendidas por um grupo à medida que foram capazes de solucionar seus problemas de adaptação externa e de integração interna, que têm funcionado bem o bastante para serem consideradas como válidas e, por essa razão, ensinadas aos novos membros como sendo o

\footnotetext{
${ }^{4}$ Especificamente atendendo a um dos objetivos da dissertação que foi associar os princípios da gestão do conhecimento à perspectiva dos processos de comunicação e, mais especificamente, à comunicação científica.
} 
modo correto de perceber, pensar e sentir em relação àqueles problemas. (Schein, 2001, p. 10).

De fato, a análise da literatura permitiu tecer argumentos para sugerir que, no contexto das organizações empresariais ou no ambiente acadêmico, é possível perceber uma estreita relação entre os processos de gestão do conhecimento, os processos de comunicação e a cultura do meio. Parece haver, portanto, uma complementaridade indissociável entre seus elementos e suas dinâmicas, conforme descrição do modelo sugerido. $\bigcirc$ primeiro argumento é que todos os fluxos de informação e conhecimento de uma determinada organização efetivam-se mediante um sistema de comunicação subjacente, o qual influencia e é influenciado pela cultura da organização. $\bigcirc$ segundo argumento é que a cultura e a comunicação, por sua vez, contribuem para criação das condições necessárias à implementação da gestão do conhecimento. $\bigcirc$ terceiro argumento, diz respeito à cultura influenciando os processos de comunicação, uma vez que ela determina hábitos, valores, normas, condutas e outros fatores. Todos estes fatores, por sua vez, influenciam diretamente os processos de comunicação. Assim, os meios utilizados, o momento adequado, a intensidade e a forma como os indivíduos se comunicam são legitimados a partir da cultura do ambiente em que elas convivem. Por seu turno, a comunicação influencia a cultura da organização, pois ela é responsável pela disseminação de valores, crenças e pressupostos, os quais permitem o questionamento, a reavaliação e o estabelecimento dos comportamentos a serem seguidos. Miller (2006) parece concordar com isso ao afirmar que a cultura organizacional é socialmente criada por meio da interação entre os membros de uma organização e, com base em outros autores, acrescenta que o estudo da cultura organizacional deve estar direcionado para a comunicação, processo por meio do qual a cultura é criada. Segundo a autora, a cultura organizacional é criada e mantida por meio de interações comunicativas entre os membros da organização. Os argumentos de Alves (1997) estão de acordo com as considerações de Miller, pois ressaltam a relevância da comunicação como um elemento de disseminação da cultura na organização. Alves lembra que a cultura é um conjunto complexo de crenças, valores, símbolos, artefatos, conhecimentos e normas, que são disseminados na organização pelos sistemas de comunicação. A comunicação, nesse sentido, é considerada um veículo na construção da cultura organizacional.

Portanto, como sugere o modelo representado na Figura 1, em uma relação mútua de influência, cultura/contexto e os processos de comunicação são responsáveis pela criação de condições necessárias para a realização dos processos de gestão do conhecimento. Isso ocorre uma vez que, numa primeira etapa, a comunicação viabiliza tanto o compartilhamento como a criação de novos conhecimentos. Como nenhum processo de criação parte do nada, mas sim daquilo que já foi anteriormente criado, criar requer, portanto, o compartilhamento de idéias, conhecimentos e informações. Para se ter acesso ao conhecimento existente (tanto tácito quanto explícito) é necessário um processo de comunicação, tanto no que diz respeito a sistemas humanos de informação, quanto aos sistemas de informação artificiais. Em uma segunda etapa, a adoção de novos modelos de gestão, tal como a gestão do conhecimento, é dependente de um processo de reavaliação da cultura organizacional. Ou seja, os valores, normas, pressupostos e crenças arraigados nas práticas organizacionais devem estar de acordo ou adequadas ao novo modelo. Assim, a cultura é responsável pela criação de um ambiente favorável ao conhecimento, um ambiente em que seja valorizado o conhecimento e seu compartilhamento. Davenport e Prusak (1999, p. 184) afirmam que uma cultura amiga do conhecimento é formada por uma série de componentes diferentes, dentre eles:

- uma orientação positiva em relação ao conhecimento: os membros da organização são inteligentes, possuem curiosidade intelectual e estão à vontade para explorar essas condições. Suas atividades de criação de conhecimento contam com a credibilidade de seus superiores:

- a ausência de inibidores do conhecimento na cultura: os indivíduos não devem ter ressentimentos em relação à organização, nem tampouco temer que o compartilhamento de seu conhecimento possa prejudicá-lo;

- o tipo de iniciativa de gestão do conhecimento deve estar de acordo com a cultura. 
Em relação ao sistema de comunicação de uma determinada organização, o conhecimento (tanto tácito como explícito) é compartilhado por meio dos canais formais e informais. A comunicação do conhecimento registrado (ou informação) dá-se principalmente a partir dos canais formais, ou seja, por meio de sistemas e mecanismos estruturados da organização. Os sistemas de informação para tomada de decisão, bases de dados, intranets, portais corporativos, dentre outros, são componentes do sistema de comunicação formal. Por meio desses sistemas estruturados, a informação pode ser processada, organizada, armazenada, recuperada e disseminada e reutilizada. É sobretudo no fluxo do conhecimento codificado que há uma incidência diversificada e mais intensa de utilização das tecnologias de informação. Por sua vez, a comunicação do conhecimento tácito dá-se principalmente por meio da interação social, a partir do processo de comunicação informal entre os indivíduos, o qual tem sido potencializado a partir da aplicação de tecnologias de comunicação. Goh (2002) parece concordar com isso ao afirmar que o conhecimento tácito é mais bem transferido por meios impessoais, fazendo uso de processos menos estruturados. $O$ autor cita alguns exemplos como a orientação, trabalho em grupo, salas de bate papo, oportunidades para conversas face a face tais como grupos de diálogo.

Ives; Torrey; Gordon (1998) sustentam parte das afirmações e das relações construídas no modelo. Os autores afirmam que a tradição oral de aprendizagem era baseada no diálogo, enquanto que na tradição escrita o aprendiz tem pouca habilidade [e possibilidade] para conversar com o criador do conhecimento, pois a criação do conhecimento é resultado da interação entre dois ou mais pontos de vista. Ives; Torrey; Gordon acrescentam que é possível que a reintrodução do diálogo, agora em níveis globais graças ao desenvolvimento e aplicação de tecnologias de comunicação, pode iniciar uma das maiores contribuições cognitivas para a atual fase da gestão do conhecimento. Em argumento semelhante, Theunissen (2004) afirma acreditar que o insucesso de projetos de gestão do conhecimento centrados nas tecnologias está direta- mente relacionado ao argumento de lves et al. (1998), de que o conhecimento é criado por meio de diálogo; ou seja, segundo Theunissen, por meio da comunicação entre dois pontos de vista.

Smoliar (2003) por seu turno, apresenta argumentos os quais, do mesmo modo, dão respaldo à construção teórica. $O$ autor argumenta que há a necessidade de mudança do termo gestão do conhecimento para gestão da interação, uma vez que o conhecimento é criado por meio da interação social, e não somente a partir de trocas de informação. Por essa razão, tornam-se imprescindíveis processos de comunicação efetivos. O autor afirma que a gestão do conhecimento tem sido gradualmente assimilada nos ambientes de trabalho, embora sua perspectiva tenda a ser limitada a modelos simplistas de trocas de informação. Smoliar (2003) ressalta ainda que, no contexto das organizações empresariais, a agitação em favor do conhecimento foi útil em seus esforços para reviver nossa consciência do significado da comunicação organizacional.

O construto teórico de Kuhlen (2003) fundamenta a reflexão e os relacionamentos presentes nos elementos do modelo: i) processos de comunicação, ii) cultura/contexto do ambiente e iii) gestão do conhecimento. $O$ autor discute duas abordagens para a gestão do conhecimento: o Knowledge Warehouse Paradigm e o Communicative Paradigm. O Knowledge Warehouse Paradigm ${ }^{5}$ estaria relacionado às abordagens de gestão do conhecimento que não levam em consideração os processos de comunicação. Assim, nessa primeira abordagem, freqüentemente o conhecimento é coletado; o conhecimento tácito é 'transformado' em explícito ao ser representado e estruturado; então, é armazenado em bases de dados ou de conhecimento; é disponibilizado por meio de linguagens tradicionais de recuperação ou ainda por meio de técnicas de mineração de dados. Por fim, o conhecimento é apresentado de maneira amigável e adaptável às necessidades de diferentes usuários, graças às flexíveis e sofisticadas formas de visualização.

No entanto, como sugere Kuhlen, um dos problemas da abordagem do Knowledge Warehouse Paradigm é que o conhecimento é recuperado fora do

\footnotetext{
${ }^{5}$ Kuhlen indica os modelos Nonaka e Takeuchi (1997), Wiig (1999) e Probst, Raub e Romhardt (2002) como representantes do Knowledge Warehouse Paradigm.
} 
seu contexto de produção. Segundo o autor, a criação do conhecimento dá-se sob circunstâncias específicas, tal como os resultados de um experimento específico, como uma generalização de dados empíricos, com aplicações específicas ou tendo em mente objetivos específicos. Dessa maneira, as circunstâncias peculiares, os fatores ambientais que o condicionaram, as características e a cultura do ambiente no qual o conhecimento é produzido, não podem ser mantidos quando este é representado em um sistema de processamento eletrônico de informação; pelo menos, não de forma adequada. $O$ ideal seria que houvesse a criação de condições que possibilitassem a junção dos recursos tangíveis de conhecimento (informação) aos especialistas, pois estes podem relacionar fragmentos de conhecimento existentes a novos contextos e novos problemas. Essa seria, em suas palavras, a mudança comunicativa (Kuhlen, 2003). Portanto, obviamente, como afirma Theunissen (2004), a visão do conhecimento socialmente construído reflete os fundamentos do processo de comunicação.

Kuhlen (2003) afirma que, com o advento das tecnologias de comunicação em grande escala, é possível observar uma mudança de paradigma: de uma visão mais estática da produção, disseminação e uso do conhecimento, para uma visão dinâmica e colaborativa desses processos, principalmente no que diz respeito à geração e trocas do conhecimento. Esta nova visão, o autor a denomina de Paradigma Comunicativo da Gestão do Conhecimento.

Na abordagem do Paradigma Comunicativo, além de ser levado em consideração o uso das fontes de informação existentes, são enfatizados os efeitos da combinação de indivíduos com diferentes backgrounds e diferentes níveis de expertise e, do mesmo modo, é enfatizada a manutenção do contexto ou a re-contextualização do conhecimento que é compartilhado. A interação social entre indivíduos, a experiência compartilhada e as trocas de conhecimento são tão importantes quanto os próprios estoques de conhecimento. Esse seria o entendimento essencial do Paradigma Comunicativo, que tem nas tecnologias de comunicação, especialmente as tecnologias de comunicação eletrônica informal (fóruns eletrônicos, comunidades virtuais e outros) uma forte aliada.
A gestão do conhecimento que é tratada neste artigo insere-se no contexto do Paradigma Comunicativo. Isto, porque considera tanto as atividades que culminam nos estoques de conhecimento, quanto os processos de comunicação que permitem as trocas informais de experiência, idéias e habilidades.

Por fim é possível sugerir que, em qualquer contexto, os processos de comunicação influenciarão e serão influenciados pela cultura do ambiente. A sugestão é um modelo em que a comunicação e a cultura são elementos que exercem influência direta sobre as ações de gestão do conhecimento. Especificamente, este modelo pode ser aplicado ao contexto do conhecimento científico, como tratado na próxima seção.

\section{Os processos de comunicação científica e a gestão do conhecimento}

O ambiente acadêmico, especialmente o das universidades, possui uma estrutura cultural, social e tecnológica constituída bastante favorável às práticas da gestão do conhecimento. Na realidade, além das universidades serem um ambiente propício, ao longo de sua existência vêm desenvolvendo e incorporando atividades e práticas de criação, disseminação e uso do conhecimento, pautadas nos processos de comunicação científica. Desse modo, tais processos são fundamentais para a gestão do conhecimento científico. Porém, como acontece de fato essa relação? O objetivo desta seção é descrever as similaridades e em que pontos os processos de comunicação científica e a gestão do conhecimento se tocam. A relação conceitual entre a comunicação científica, cultura científica e a gestão do conhecimento, podem ser descritos no modelo ilustrado na Figura 2. O modelo, aplicado ao contexto do conhecimento científico, pressupõe os mesmos relacionamentos que os descritos na seção anterior. $A$ teoria embutida no modelo sugere que os processos de comunicação científica, a cultura do ambiente acadêmico e a gestão do conhecimento possuem um estreito e indissociável relacionamento. 


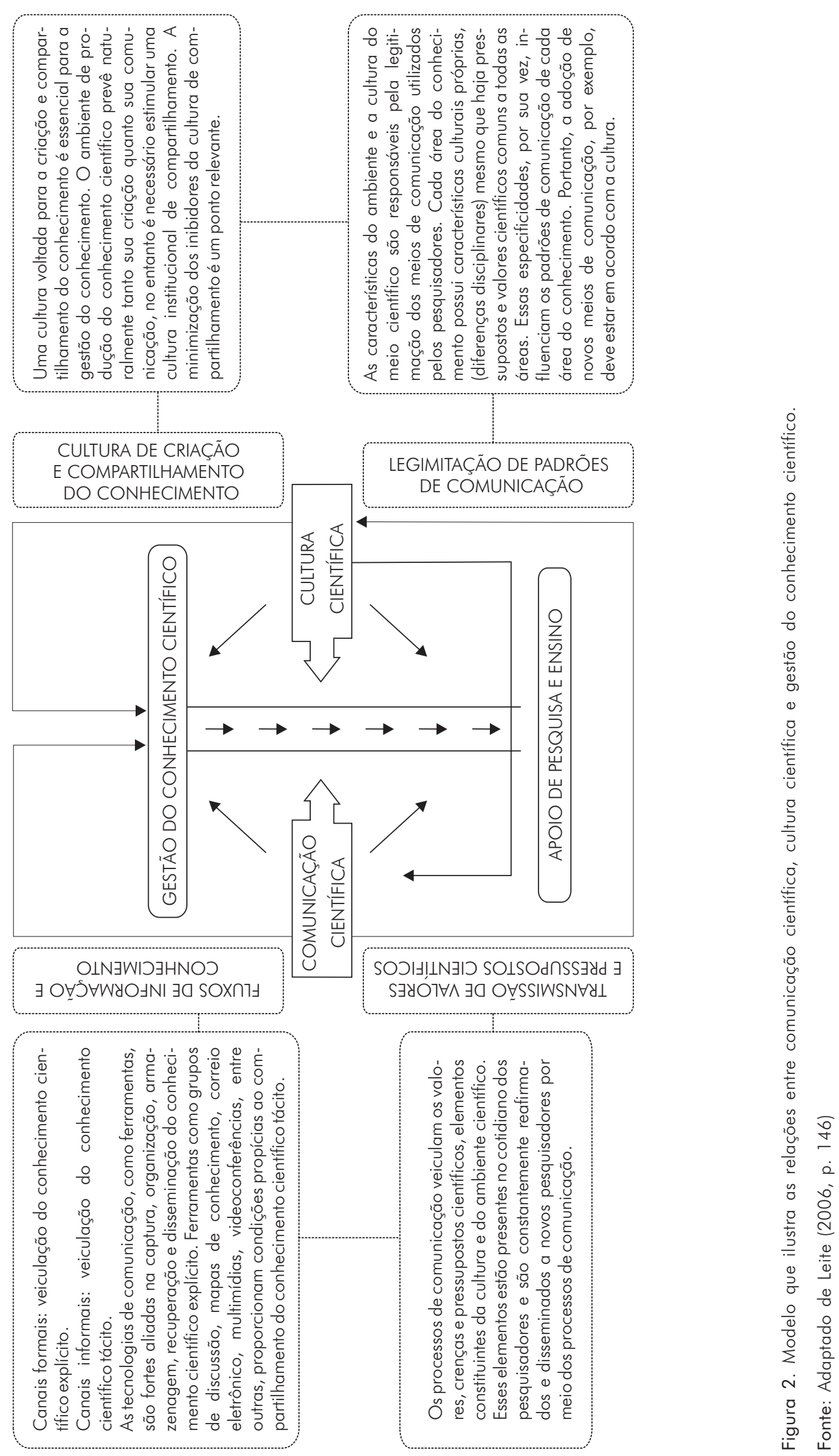

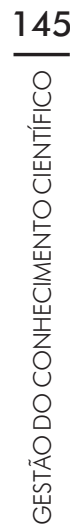

TransInformação, Campinas, 19(2):139-151, maio/ago., 2007 
Ainda atendendo a um dos objetivos da pesquisa, destacam-se a seguir as similaridades e/ou complementaridades existentes entre os processos de comunicação científica e as práticas de gestão do conhecimento.

O compartilhamento e a disseminação - isto é, a comunicação do conhecimento científico - são processos fundamentais para a continuidade e o avanço da ciência. Como observa Crane (1972), o processo de comunicação científica é uma espécie de difusão de idéias transmitidas de pessoa a pessoa, paralelamente a um processo de interação social, o qual sublinha o desenvolvimento do conhecimento científico. Shaughnessy (1989), por seu turno, define a comunicação científica como sendo um fenômeno social segundo o qual a atividade intelectual e criativa é passada de um cientista para outro. Assim, a comunicação científica é responsável tanto pelo compartilhamento do conhecimento científico registrado - a informação científica - quanto pelo compartilhamento do conhecimento científico relacionado à produção, experiências e habilidades dos cientistas, compartilhadas informalmente. Kaplan e Storer (1968) afirmam que a comunicação científica refere-se às trocas de informação e idéias entre cientistas nas suas funções como cientistas. $\mathrm{Na}$ definição dos autores, há a distinção entre informação, o registro do conhecimento científico, e idéias, esta talvez relacionada ao conhecimento que não é 'explicitável' em publicações científicas. Menzel (1966 apud Kaplan e Storer, 1968, p.112) define a comunicação científica como a totalidade de publicações, facilidades, ocasiões, arranjos institucionais e costumes, que afetam direta ou indiretamente a transmissão de mensagens científicas entre cientistas. As definições de comunicação científica destacadas, ressaltam conceitos e aspectos de interesse para a gestão do conhecimento, como, por exemplo, o compartilhamento da atividade intelectual e criativa, trocas de informação e idéias, publicações, facilidades, ocasiões. Todos esses aspectos são de interesse para a visualização das similaridades entre a comunicação científica e a gestão do conhecimento.

A comunicação científica - entendida neste artigo como todas as formas de facilitação, interação e trocas de informação, conhecimento, experiências e habilidades entre os membros das comunidades científicas - possui funções específicas. Obviamente, as funções e atores do sistema de comunicação científica são transformados ou modificados ao longo do tempo, principalmente por influência do desenvolvi- mento de tecnologias de informação e comunicação. Kaplan e Storer (1968, p. 1 12), com base em Menzel (1966) descrevem as principais funções da comunicação científica, que são:

- prover ao cientista as melhores respostas a questões específicas;

- contribuir para que o cientista esteja ciente de novos desenvolvimentos em seu campo de atuação;

- estimular o cientista a buscar novos conhecimentos além de suas áreas de interesses;

- divulgação das principais tendências de áreas emergentes, fornecendo aos cientistas idéia da relevância de seu trabalho;

- testar a confiabilidade de novos conhecimentos, diante da possibilidade de testemunhos e de verificações;

- redirecionar ou ampliar o rol de interesse dos cientistas,

- fornecer feedback para o aperfeiçoamento da produção do pesquisador.

As funções atribuídas por Menzel podem ser consideradas como boas práticas e objetivos em relação ao conhecimento, para o desenvolvimento da gestão do conhecimento científico. Roosendaal e Geurts (1998) também definiram as funções mais familiares da comunicação do conhecimento científico, indicando o que se considera neste artigo como condições férteis para a sua gestão. As funções são:

- o registro da autoria que assegura o reconhecimento e a prioridade na propriedade sobre um determinado avanço ou descoberta científica;

- a certificação, que permite serem assegurados o controle da qualidade e a validade de determinado conhecimento, por meio do processo de avaliação pelos pares;

- awareness (estar ciente, alerta), que possibilita a disseminação e acessibilidade às pesquisas e que os pares de uma determinada comuni-dade científica estejam cientes ou atentos a novas descobertas; para tanto, os resultados de pesquisas são comunicados por meio de livros e artigos em conferências e periódicos, àqueles que partilham o interesse pelo mesmo tópico;

- o armazenamento, que guarda e preserva o registro do conhecimento científico por muito 
tempo. Os autores observam que editores e bibliotecas estão criando repositórios eletrônicos de informação e permitem a distribuição desse acervo por uma grande variedade de meios eletrônicos.

No que concerne à gestão do conhecimento, a literatura descreve etapas ou processos que constituem modelos que a representam. Dessa forma, a análise da literatura, principalmente o estudo realizado por Stollenwerk (2001), que sintetiza de modo interessante parte dos modelos encontrados na literatura, são úteis para sugerir que identificação, captura ou aquisição, validação, organização e armazenagem compartilhamento/disseminação e criação do conhecimento constituem os processos básicos da gestão do conhecimento. Entende-se que a gestão do conhecimento está relacionada com a criação de condições férteis, a condução de situações ótimas, viabilizadoras para que o conhecimento seja criado, compartilhado, assimilado e convertido em benefícios aplicáveis à consecução dos objetivos de uma determinada organização.

Assim, considerando-se que a gestão do conhecimento não será viabilizada sem que processos de comunicação efetivos ocorram, conforme o modelo, sugere-se que a gestão do conhecimento científico, no contexto acadêmico, está intimamente ligada aos processos de comunicação científica. Como resultado, e fundamentado i) nas descrições das funções ou processos de comunicação científica e dos processos básicos de gestão do conhecimento realizada na análise da literatura, e ii) nas reflexões construídas ao longo do trabalho, é possível apontar similaridades entre as duas abordagens, conforme o Quadro 1.

Quadro 1. Similaridades entre processos de gestão do conhecimento e a comunicação científica.

\begin{tabular}{|c|c|}
\hline GESTÃO DO CONHECIMENTO ${ }^{1}$ & COMUNICAÇÃO CIENTÍFICA \\
\hline \multicolumn{2}{|l|}{ Identificação } \\
\hline $\begin{array}{l}\text { Mapeamento do conhecimento. Levantamento de informa- } \\
\text { ções e conhecimentos que são criados e usados no ambiente } \\
\text { da organização. Identificação de competências críticas para } \\
\text { o sucesso da organização. Cada competência individual } \\
\text { remete à areas de conhecimento que as sustentam. Iden- } \\
\text { tificação de fontes internas e externas de conhecimento. }\end{array}$ & $\begin{array}{l}\text { Os levantamentos bibliográficos realizados por pesquisadores } \\
\text { podem, de certa forma, estar relacionados ao mapeamento } \\
\text { do conhecimento e à identificação de fontes de informação } \\
\text { científica. Além disso, bibliotecas acadêmicas contribuem } \\
\text { formalmente para a identificação de conhecimentos na } \\
\text { medida em que oferecem serviços relacionados em meio } \\
\text { tradicional e eletrônico. }\end{array}$ \\
\hline
\end{tabular}

Captura/Aquisição

Processo de aquisição de informação, conhecimento, experiências, habilidades que serão necessárias para proporcionar a criação e a manutenção das competências essenciais e áreas do conhecimento identificadas e mapeadas. Relacionado também à importação de uma parte de conhecimento de fontes externas à organização, como o conhecimento proveniente das relações com os clientes, fornecedores, concorrentes, parceiros e outros.
As universidades, como celeiros da produção do conhecimento científico, possuem a maior concentração de pesquisadores de alto nível que estão constantemente capturando conhecimento tanto formalmente, nos principais periódicos, repositórios, livros, e outras fontes, quanto informalmente, em conferências nacionais e internacionais, contatos pessoais com outros pesquisadores e pesquisadores renomados, fluxo de informação e conhecimento nos colégios invisíveis, visitas a outras instituições e laboratórios realização de estágios pós-doutorais em instituições estrangeiras e outros. Por outro lado, as bibliotecas acadêmicas ou universitárias possuem, como serviço-meio, atividades tradicionais de aquisição de livros, periódicos e outros documentos científicos, com base no perfil e demanda de sua comunidade usuária.

\footnotetext{
1 A descrição de cada conceito foi retirada dos seguintes autores: Probst et al. (2002), Despres e Chauvel (1999), Wiig (2002) e Stollenwerk (2001).
} 
continuação quadro 1 .

\begin{tabular}{ll}
\hline GESTÃO DO CONHECIMENTO & COMUNICAÇÃO CIENTÍFICA \\
\hline
\end{tabular}

Validação

Atividade com o fim de filtrar o conhecimento, avaliar sua qualidade, confiabilidade, sintetizá-lo para fins de aplicação em um outro momento.

No que diz respeito ao sistema de comunicação formal, por meio do sistema de avaliação pelos pares e pelo processo editorial, o conhecimento é avaliado e validado. Da mesma forma, em relação aos meios informais de comunicação, os pesquisadores carregam consigo o receio de tornar público um determinado conhecimento que não esteja minimamente fundamentado em argumentos confiáveis da literatura ou da percepção proveniente da experiência. Portanto, o próprio sistema científico se encarrega, naturalmente, em manter padrões de qualidade e confiabilidade ao conhecimento científico produzido e compartilhado.

\section{Organização/Armazenagem}

Tem por objetivo a garantia da recuperação rápida, fácil e correta do conhecimento por meio de sistemas efetivos. As etapas desse processo compreendem a classificação do conhecimento validado; definição da arquitetura de tecnologias a serem utilizadas; criação e gerenciamento de bancos de dados, informações e conhecimentos. Indivíduos e organizações estocam informação em sistemas de memória de vários tipos, como no cérebro, em discos rígidos, arquivos, bibliotecas e armazém de dados.

\section{Compartilhamento/disseminação}

Pressuposto básico e primordial para a transformação de informações e experiências isoladas em algo que toda a organização possa utilizar. Diz respeito ao processo de compartilhamento e disseminação do conhecimento que já está na organização. Compreende questões importantes em todo o processo tais como distribuição do conhecimento de forma seletiva, ao maior número de usuários possível, em tempo hábil e local apropriado. A utilização de tecnologias se dá com maior intensidade nesse processo.

\section{Criação}

Relacionado à criação de novas habilidades, novos produtos, idéias melhores e processos mais eficientes. Inclui esforços propositados para criar capacidades as quais ainda não se encontram presentes na organização. O conhecimento é desenvolvido por meio da aprendizagem, inovação, criatividade e da importação de conhecimento do ambiente externo à organização. Este processo envolve as seguintes dimensões: a aprendizagem, externalização, lições aprendidas, criatividade, pesquisa, experimentação, descoberta e inovação.
As bibliotecas são as principais instâncias onde os registros do conhecimento científico são organizados e armazenados. Com o desenvolvimento e aplicação de tecnologias de comunicação alternativas como os repositórios institucionais, repositórios temáticos e periódicos científicos eletrônicos baseados no OAI-PMH que permitem maior efetividade na organização, armazenagem e compartilhamento da informação.
O conhecimento científico é compartilhado por meios de comunicação formais e informais. O sistema de comunicação formal veicula os registros do conhecimento por meio de livros, periódicos científicos, relatórios de pesquisa, anais de conferências, teses, dissertações e outros. Esses meios lidam especialmente com o conhecimento científico explícito, e possuem, sobretudo por conta de desenvolvimento de tecnologias de informação, um público amplo, tanto da instituição quanto da comunidade científica. Estão nesse contexto os serviços de informação, bibliotecas, serviços de disseminação seletiva, bibliotecas digitais, repositórios digitais e outros. O sistema de comunicação informal compartilha também conhecimento explícito, porém lida com parte do conhecimento científico tácito possível de ser compartilhado. O conhecimento nesse caso é compartilhado por meio das atividades de ensino, tanto em nível de graduação quanto pós-graduação, reuniões de grupos de pesquisa, reuniões de orientação de alunos de doutorado, mestrado e iniciação científica, conferências, seminários, contatos pessoais na própria instituição, contatos informais com membros da mesma comunidade científica, interação nos colégios invisívies, grupos de discussão, ambientes de aprendizagem não presencial e outros.
O processo de criação do conhecimento científico dá-se por meio da realização de pesquisas científicas, geralmente realizadas em grupos de pesquisa. Além disso, o conhecimento é criado também por meio da formação de pesquisadores em nível de pós-graduação. Nesse sentido, os programas de pós-graduação constituem a principal fonte de criação do conhecimento, capacidades, competências e habilidades relacioandas à ciência.

Fonte: Leite (2006, p. 149).

TransInformação, Campinas, 19(2):139-151, maio/ago., 2007 
Por outro lado, no que diz respeito especificamente à criação do conhecimento científico, observase também que os processos de comunicação científica contribuem para a realização dos modos de conversão de Nonaka e Takeuchi (1997). Sabe-se, porém, que a teoria dos modos de conversão está relacionada à perspectiva da criação do conhecimento organizacional. Contudo, respeitando suas especi-ficidades, é possível

Quadro 2. Similaridades entre os modos de conversão e a comunicação científica.

\begin{tabular}{ll}
\hline MODO DE CONVERSÃO & COMUNICAÇÃO CIENTÍFICA \\
\hline
\end{tabular}

Socialização

Transformação do conhecimento tácito em conhecimento tácito
Os canais de comunicação científica informais proporcionam os meios para que parte do conhecimento científico tácito de um determinado pesquisador seja comunicado e transformado em conhecimento científico tácito de outro pesquisador. As comunidades científicas, por meio da interação social entre pesquisadores nos colégios invisíveis, são um dos meios para que isso ocorra. $O$ intercâmbio de pesquisadores de diferentes instituições, contatos informais em congressos, seminários costumam veicular támbém conhecimento tácito. As atividades científicas como reunião de grupos de pesquisa, reunião de orientação de alunos, ensino e vivência em laboratórios costumam ser meios privilegiados de compartilhamento do conhecimento científico tácito.

\section{Externalização}

Transformação do conhecimento tácito em conhecimento explícito
As publicações científicas (artigos, pré-printes, livros, teses, dissertações, trabalhos de congressos, textos para discussão, relatórios de pesquisa e outros) são resultado da externalização da experiência adquirida, das habilidades e do conhecimento desenvolvido e adquirido durante as atividades científicas do pesquisador. Ou seja, o pesquisador isola parte de sua estrutura cognitiva e o transforma em uma estrutura comunicável - em conhecimento explícito. Esse conhecimento explícito, a informação, é armazenado, recuperado e veiculado principalmente por meio dos canais formais de comunicação.

\section{Internalização}

Transformação do conhecimento explícito em conhecimento tácito
Os registros do conhecimento científico, a informação científica, para existir como tal, necessita ser comunicada. Os meios para que isso ocorra são estruturados de tal modo que permita sua organização, armazenagem, recuperação e disseminação. Dessa forma, as bibliotecas, as bibliotecas digitais, portais, repositórios e serviços de informação dispõem de meios para que a informação científica certa, no formato adequado, no momento certo chegue à pessoa certa. Assim, todo esse sistema funciona para que um determinado conhecimento científico explícito comunicado por um pesquisador seja utilizado e internalizado, para então passar a constituir a estrutura cognitiva de um outro pesquisador. Esse processo pode ser percebido no estágio de formulação do problema de pesquisa, no momento em que o pesquisador busca fontes de informação e analisa a literatura científica tanto da sua área como de áreas correlatas. Um dos objetivos desse processo é aprender mais sobre o tópico específico. Nesse instante há a internalização de conhecimento científico explícito.

\section{Combinação}

Transformação do conhecimento explícito em conhecimento explícito
O sistema de comunicação científica formal provê condições para que um pesquisador tenha acesso à literatura científica que ele necessita, ou seja, que tenha acesso ao conhecimento científico explícito. As informações que um determinado pesquisador acessa serão combinadas, reagrupadas, reorganizadas de forma a agregar valor e acrescentar ao novo conjunto de informações um novo conhecimento explícito, gerando um novo conhecimento. É o que acontece com as revisões de literatura, por exemplo. De outro modo, as informações coletadas durante a realização de uma pesquisa podem passar por um rearranjo e serem utilizadas em outra situação ou pesquisa.

Fonte: Leite (2006, p. 151) 
extrapolar a contribuição e descre-ver os seus processos no contexto da criação do conhecimento científico com base em processos de comunicação científica. Desse modo, a análise da literatura sobre processos de comunicação científica e gestão do conhecimento permitiu identificar indícios de que os modos de conversão, socialização - externalização, combinação e internalização, no contexto e na criação do conhecimento científico, apoiam-se em processos de comunicação científica conforme as similaridades apontadas no Quadro 2.

Ao serem evidenciadas as similaridades entre os processos da gestão do conhecimento e os processos ou funções da comunicação científica, faz-se necessário tecer algumas considerações acerca da complementaridade e interdependência entre as duas abordagens. Conforme mencionado anteriormente, as universidades, ao longo de sua existência, desenvolveram e incorporaram práticas voltadas para a criação, disseminação e uso do conhecimento científico pautadas nos processos de comunicação realizados, especialmente, no âmbito das comunidades científicas. No entanto, tais práticas não dispõem, ainda, de uma sistematização apropriada para que seja obtido o máximo benefício dos recursos empregados, do conhecimento produzido a partir de suas atividades e nem tão pouco da expertise desenvolvida por seus pesquisadores. A contribuição da gestão do conhecimento nesse contexto está na possibilidade de maximizar o potencial, por meio, obviamente, do gerenciamento de recursos tangíveis de conhecimento, aliado à promoção de condições oportunas e férteis para a sua criação, compartilhamento (formal e informalmente) e uso efetivos. Na medida em que é realizada a gestão do conhecimento, os processos de comunicação tornam-se mais eficientes e, sendo mais eficientes, prorpocionam a gestão do conhecimento com maior sucesso. Por fim, a gestão do conhecimento científico diz respeito ao planejamento e controle de ações (políticas, mecanismos, ferramentas, estratégias e outros) que governam o fluxo do conhecimento científico em suas vertentes tácita e explícita, tendo como substrato os processos de comunicação científica com a finalidade de apoiar e maximizar a geração de novos conhecimentos e o ensino.

\section{CONSIDERAÇÕES FINAIS}

Estudos e aplicações de gestão do conhecimento devem considerar as especificidades do contexto no qual serão desenvolvidos, a natureza do processo de criação do conhecimento e, sobretudo, não devem prescindir dos processos de comunicação próprios do ambiente. É contra-producente mencionar processos como criação e compartilhamento do conhecimento científico (fases da gestão do conhecimento científico) sem necessariamente considerar a comunicação que contribui e torna viável tais processos. Desse modo, a comunicação científica constitui uma camada essencial da gestão do conhecimento científico no contexto de universidades.

Embora as construções teóricas realizadas neste artigo tentem ser amplas o suficiente para contemplar aspectos importantes relacionados com especificidades e diferenças disciplinares entre áreas do conhecimento, há a necessidade de se estudar como essas diferenças influenciariam a gestão do conhecimento científico em diferentes áreas do saber. É relevante considerar essa variável, pois sabe-se que os padrões de comunicação de pesquisadores variam de acordo com a área do conhecimento, por conseqüência, há necessidade de modelos de gestão do conhecimento científico para cada área ou grande área.

A evidenciação das similaridades entre a comunicação científica e a gestão do conhecimento, enfatiza a comunicação científica como um elemento inexorável e indispensável à gestão do conhecimento científico. A formulação conceitual proposta neste artigo, visou à contribuição para o quadro teórico de referência, necessário à consolidação de estudos, apropriadamente fundamentados e sob a luz da Ciência da Informação, sobre a gestão do conhecimento científico no contexto de universidades.

\section{REFERÊNCIAS}

ALVES, S. Revigorando a cultura da empresa: uma abordagem cultural da mudança nas organizações na era da globalização. São Paulo: Makron Books, 1997.

ASH, J. Communication missing from KM's core strategies. 2000. Disponível em: <http://www.knowledgepoint.com.au/
knowledge_management/Articles/KM_JA001.htm > . Acesso em: 23 out. $20 \overline{0} 5$.

CRANE, D. Invisible colleges: Diffusion of knowledge in scientific communities. Chicago, London: University of Chicago Press, 1972. $213 p$. 
DAVENPORT, T., PRUSAK, L. Conhecimento empresarial. Rio de Janeiro: Campus, 1999. 237p.

DESPRES, C., CHAUVEL, D. Knowledge management(s). Journal of Knowledge Management. v.3, n.2, p.110-120, 1999. Disponível em: <http://www.emeraldinsight.com/Insight/html/ Output/Published/EmeraldFullTextArticle/Articles/ 2300030202.html>. Acesso em: 3 dez. 2004.

$\mathrm{GOH}$, S.C. Managing effective knowledge transfer: an integrative framework and some practice implications. Journal of Knowledge Management, v.6, n.1, p.23-30. 2002. Disponível em: <www.emeraldinsight.com/10.1108/13673270210417664>. Acesso em: 8 set. 2005.

IVES, W.; TORREY, B.; GORDON, C. Knowledge management: An emerging discipline with a long history. Journal of Knowledge Management, v. 1, n.4, p.269-274, 1998. Disponível em: <http:/ /www.krii.com/downloads/km_emerg_discipl.pdf $>$. Acesso em: 12 jan. 2006

JENSEN, B. Communication or knowledge management?: it's time to wake up and smell the koffee. Communication World, 1998. Disponível em: <http://www.simplerwork.com/library/ w2.htm >. Acesso em: 18 set. 2005.

KAPLAN, N.; STORER, N.W. Scientific communication. In: SILLS, D.L. International encyclopedia of the social sciences. New York: Macmillan, v.14, p.112-117, 1968.

KUHLEN, R. Change of paradigm in knowledge management: framework for the collaborative production and exchange of knowledge. In: $\mathrm{HOBOHM}, \mathrm{H}$. (ed.). Knowledge management: libraries and librarians taking up the challenge. MÜNCHEN: K.G. Saur, 2003, p.21-38. (IFLA Publications) Disponível em: $<$ http://www.inf-wiss.uni-konstanz.de/People/ RK/Vortraege03Web/rk ifla03 for publ300803.pdf>. Acesso em: 4 dez. 2005.

LEITE, F.C.L. Gestão do conhecimento científico no contexto acadêmico: proposta de um modelo conceitual. 2006. Dissertação (Mestrado em Ciência da Informação) - Universidade de Brasília. Brasília. Disponível em: <http://eprints.rclis.org/ archive/00006259/>. Acesso em: 3 jan. 2007.

MARTENSSON, M. A critical review of knowledge management as a management tool. Journal of Knowledge Management, v.4, n.3, p.204-216, 2000. Disponível em: <http://www. emeraldinsight.com/10.1 108/13673270010350002 >. Acesso em: 12 out. 2004.
MENZEL, H. Scientific communication: five themes from social science research. American Psychologist, v.21, n.10, p.999-1004, 1966.

MILLER, K. Organizational communication: Approaches and processes. 4.ed. Belmont. CA: Thomson Wadsworth, 2006. 364p. NONAKA, I.; TAKEUCHI, H. Criação do conhecimento na empresa: como as empresas japonesas geram a dinâmica da inovação. Rio de Janeiro: Campus, 1997. 358p.

PROBST, G.; RAUB, S.; ROMHART, K. Gestão do conhecimento: os elementos construtivos do sucesso. Porto Alegre: Bookman, 2002. 286p.

ROOSENDAAL, H.E.; GEURTS, P.A.T.M. Forces and functions in scientific communication: An analysis of their interplay. 1998. Disponível em: <http://www.physik.uni-oldenburg.de/ conferences/crisp97/roosendaal.html>. Acesso em: 25 jan. 2006.

SCHEIN, E.H. Guia de sobrevivência da cultura corporativa. Rio de Janeiro: José Olympio, 2001. 192p.

SHARP, D. Knowledge management today: Challenges and opportunities. Information Processing and Management, v. 20, n. 2, p. 32-37, 2003.

SHAUGNESSY, T.W. 'Scholarly communication: The need for an agenda for action - A symposium. The Journal of Academic Librarianship, v. 15, n.2, p.68, 1989.

SMOLIAR, S.W. Interaction management: The next (and necessary) step beyond knowledge management. Business Process Management Journal. v.9, n.3, p.337-353, 2003. Disponível em: <http://www.emeraldinsight.com/10.1108/1463715 0310477920 >. Acesso em: 22 jan. 2006.

STOLLENWERK, M.F.L. Gestão do conhecimento: conceitos e modelos. In: TARAPANOFF, K. Inteligência organizacional e competitiva. Brasília: Universidade de Brasília, 2001. 344p.

THEUNISSEN, P. Communication: The cornerstone of knowledge management. Making a difference: Australian and New Zealand Communication Association Conference, 2004.

WIIG, K.M. Comprehensive Knowledge Management. Knowledge Research Institute, Arlington, TX. 1999. Disponível em: <http:// www.krii.com/downloads/comprehensive_km.pdf $>$. Acesso em: 23 out. 2005

WIIG, K.M. Knowledge management has many facets. 2002. Disponível em: <http://www.krii.com/downloads/Four_KM_ Facets.pdf > . Acesso em: 5 abr. 2005. 
